\title{
AN EXPLANATION FOR THE OBSERVED WEAK SIZE EVOLUTION OF DISK GALAXIES
}

\author{
Rachel S. Somerville, ${ }^{1}$ Marco Barden, ${ }^{1}$ Hans-Walter Rix, ${ }^{1}$ Eric F. Bell, ${ }^{1}$ Steven V. W. Beckwith, ${ }^{2,3}$ \\ Andrea Borch, ${ }^{1,4}$ John A. R. Caldwell, ${ }^{5}$ Boris Häußler, ${ }^{1}$ Catherine Heymans, ${ }^{6}$ Knud JahnKe, ${ }^{1}$ \\ Shardha Jogee, ${ }^{7}$ Daniel H. McIntosh, ${ }^{8}$ Klaus Meisenheimer, ${ }^{1}$ Chien Y. Peng, ${ }^{2}$ \\ Sebastian F. Sánchez, ${ }^{9}$ Lutz Wisotzki, ${ }^{10}$ and Christian Wolf ${ }^{11}$ \\ Received 2006 December 15; accepted 2007 September 2
}

\begin{abstract}
Surveys of distant galaxies with the Hubble Space Telescope and from the ground have shown that there is only mild evolution in the relationship between radial size and stellar mass for galactic disks from $z \sim 1$ to the present day. Using a sample of nearby disk-dominated galaxies from the Sloan Digital Sky Survey (SDSS) and high-redshift data from the GEMS (Galaxy Evolution from Morphology and SEDs) survey, we investigate whether this result is consistent with theoretical expectations within the hierarchical paradigm of structure formation. The relationship between virial radius and mass for dark matter halos in the $\Lambda$ CDM model evolves by about a factor of 2 over this interval. However, $N$-body simulations have shown that halos of a given mass have less centrally concentrated mass profiles at high redshift. When we compute the expected disk size-stellar mass distribution, accounting for this evolution in the internal structure of dark matter halos and the adiabatic contraction of the dark matter by the selfgravity of the collapsing baryons, we find that the predicted evolution in the mean size at fixed stellar mass since $z \sim 1$ is about $15 \%-20 \%$, in good agreement with the observational constraints from GEMS. At redshift $z \sim 2$, the model predicts that disks at fixed stellar mass were on average only $60 \%$ as large as they are today. Similarly, we predict that the rotation velocity at a given stellar mass (essentially the zero point of the Tully-Fisher relation) is only about $10 \%$ larger at $z \sim 1(20 \%$ at $z \sim 2)$ than at the present day.
\end{abstract}

Subject headings: cosmology: observations — galaxies: evolution — galaxies: high-redshift — galaxies: spiral - surveys

Online material: color figures

\section{INTRODUCTION}

The relationship between the radial size and the luminosity or stellar mass of galactic disks is a fundamental scaling relation that reveals important aspects of the formation history of these objects. The size, luminosity/mass, and rotation velocity form a "fundamental plane" for disks at the present epoch (Pizagno et al. 2007) that is analogous to the more familiar fundamental plane for early-type galaxies (Burstein et al. 1997). The zero point, slope, and scatter of the fundamental plane for both disks and spheroids, and the evolution of these quantities over cosmic time, pose strong constraints on models of galaxy formation.

In the modern paradigm of disk formation, cold dark matter $(\mathrm{CDM})$ dominates the initial gravitational potential, and dark matter (DM) and gas acquire angular momentum via tidal tor-

\footnotetext{
1 Max-Planck-Institut für Astronomie, Königstuhl 17, Heidelberg, 69117, Germany.

${ }^{2}$ Space Telescope Science Institute, 3700 San Martin Drive, Baltimore, MD 21218.

3 Johns Hopkins University, 3400 North Charles Street, Baltimore, MD 21218.

4 Astronomisches Rechen-Institut, Mönchhofstrasse 12-14, D-69120, Heidelberg, Germany.

5 University of Texas, McDonald Observatory, Fort Davis, TX 79734.

6 Department of Physics and Astronomy, University of British Columbia, 6224 Agricultural Road, Vancouver, BC V6T 1Z1, Canada.

7 Department of Astronomy, University of Texas at Austin, 1 University Station, C1400, Austin, TX 78712-0259.

8 Department of Astronomy, University of Massachusetts, 710 North Pleasant Street, Amherst, MA 01003.

9 Centro Hispano Aleman de Calar Alto, C/Jesus Durban Remon 2-2, Almeria, E-04004, Spain.

${ }^{10}$ Astrophysikalisches Institut Potsdam, An der Sternwarte 16, Potsdam, 14482, Germany.

11 Department of Physics, Denys Wilkinson Building, University of Oxford, Keble Road, Oxford, OX1 3RH, UK.
}

ques in the early universe (Peebles 1969). When the gas cools and condenses, this angular momentum may eventually halt the collapse and lead to the formation of a rotationally supported disk (Fall \& Efstathiou 1980). Under the assumption that the specific angular momentum of the precollapse gas is similar to that of the $\mathrm{DM}$ and is mostly conserved during collapse, this picture leads to predictions of present-day disk sizes that are in reasonably good agreement with observations (Kauffmann 1996; Dalcanton et al. 1997; Mo et al. 1998; Avila-Reese et al. 1998; Somerville \& Primack 1999; van den Bosch 2000; Dutton et al. 2007; Gnedin et al. 2006).

However, in the most sophisticated numerical hydrodynamic simulations of disk formation in a CDM universe, the protodisk gas tends to lose a large fraction of its angular momentum via mergers, leading to disks that are too small and compact (Navarro \& White 1994; Sommer-Larsen et al. 1999; Navarro \& Steinmetz 2000). It is still not clear whether this problem reflects a fundamental difficulty with CDM (i.e., excess small-scale power) or is due to inadequate numerical resolution or treatment of "gastrophysical" processes such as star formation and feedback (Governato et al. 2004; Robertson et al. 2004). However, it has been suggested that delayed cooling and star formation, perhaps due to strong feedback in low-mass progenitors, could help to stem this angular momentum loss (Weil et al. 1998; Maller \& Dekel 2002). These ideas can be tested by observing the evolution of disk scaling relations at high redshift relative to the present epoch.

The observational relationship between radial size (effective radius or disk scale length) and luminosity or stellar mass for disks at low redshift has now been well characterized by studies based on the Sloan Digital Sky Survey (SDSS; e.g., Shen et al. 2003, hereafter S03). Several pioneering studies in the past decade studied the size-luminosity relation for disks out to 
redshift $z \sim 1$ (e.g., Lilly et al. 1998; Simard et al. 1999). Sizes were also measured for Lyman break galaxies (LBGs) at redshifts $z \sim 3$ (Giavalisco et al. 1996; Lowenthal et al. 1997). However, there was significant disagreement between the results of different studies, and a clear understanding of the evolution of galaxy sizes over cosmic time was hampered by the difficulty of obtaining large samples with accurate redshifts and highresolution imaging, and by concerns about the impact of surface brightness selection effects. An additional problem with interpreting high-redshift data is that as higher redshifts are probed, the observed optical begins to shift into the rest-frame UV, and $k$-corrections become highly uncertain. Moreover, the stellar mass-to-light ratios of galaxies increase as their stellar populations age, and therefore the size-luminosity relation would change over time even for galaxies that were just "passively" aging.

Recently, new studies with the Advanced Camera for Surveys (ACS) on board the Hubble Space Telescope (HST) have provided greatly improved constraints on the disk size-luminosity relation at high redshift. Ravindranath et al. (2004) and Ferguson et al. (2004) presented size distributions for $z \lesssim 1$ disk-type galaxies and for rest-frame UV selected galaxies from $1.4 \lesssim z \lesssim 6$, respectively, based on samples selected from the Great Observatories Origins Deep Survey (GOODS). Barden et al. (2005, hereafter B05) presented the luminosity-size and stellar mass-size relation out to $z \sim 1$ based on the GEMS (Galaxy Evolution from Morphology and SEDs) survey (Rix et al. 2004). They concluded that disk galaxies of a given size at $z \sim 1$ are $\sim 1$ mag brighter in the $V$ band, but that there is less than about a $10 \%$ change in the stellar mass at a given size between $z \sim 1$ and the present. This result is consistent with a mean stellar mass-to-light ratio that increases with time, as expected based on the simple aging of stellar populations. An interesting complementary result was recently found by Sargent et al. (2007) based on the COSMOS survey. They found that the number density of disks with half-light radii between 5 and $7 \mathrm{kpc}$ is nearly constant from $z \sim 1$ until the present. Coupled with findings that the stellar mass function of spiral galaxies at $z \sim 1$ was about the same as it is today (Borch et al. 2006), this reinforces a picture in which disks have a fixed relationship between their stellar mass and their radial size over this redshift interval (i.e., that as disks grow in mass through star formation, their sizes grow in such a way as to keep them on this relation, on average).

Trujillo et al. (2004) measured luminosity-size and stellar mass-size relations in the rest-frame optical (based on the groundbased near-infrared selected FIRES [Faint Infrared Extragalactic Survey] sample) out to $z \sim 2.5$ and found that the average surface brightness at $z \sim 2.5$ is about $2-3$ mag $\operatorname{arcsec}^{-1}$ brighter than in the local universe, but the average size at a fixed stellar mass has evolved by less than a factor of 2 . Trujillo et al. (2006, hereafter T06) presented the results of a similar analysis of a larger sample from FIRES and combined those results with the lower redshift studies of S03 and B05.

How do these observations compare with theoretical expectations? In the simplest version of the Fall-Efstathiou picture, under the assumption that halo mass density profiles are singular isothermal spheres [SIS; $\rho(r) \propto 1 / r^{2}$ ] and neglecting the selfgravity of the baryons, we expect the size of a galactic disk that forms within a dark matter halo to scale as $r_{\text {disk }} \propto \lambda r_{\text {vir }}$, where $\lambda$ is the dimensionless spin parameter and $r_{\mathrm{vir}}$ is the virial radius of the dark matter halo. $N$-body simulations have demonstrated that the spins of dark matter halos are not correlated with halo mass or most other properties, and the distribution does not evolve with time (e.g., Bullock et al. 2001a). If the stellar mass of the disk is a constant fraction of the halo virial mass, then in this simple pic- ture, the expectation is that the average size of galactic disks of a given stellar mass will evolve as $r_{\text {vir }}$ evolves for halos of a given virial mass. In the currently favored $\Lambda$ CDM cosmology, this would imply a decrease of a factor of $\sim 1.7$ out to $z=1$ and a factor of $\sim 3$ out to $z \sim 3$. This $r_{\text {disk }} \propto \lambda r_{\text {vir }}$ scaling (hereafter referred to as the SIS model) has frequently been used in the literature as a theoretical baseline (e.g., Mao et al. 1998; Ferguson et al. 2004 B05; T06). Mao et al. (1998) found that the SIS model was consistent with the size evolution of disks out to $z \sim 1$ compared with the data available at the time, but the samples were tiny, and the observational selection effects were not well characterized or accounted for. Ferguson et al. (2004) also found that the average rest-frame UV sizes of restframe UV selected galaxies at $1.4 \lesssim z \lesssim 5$ were consistent with the SIS model, but the connection of rest-frame UV luminosity with stellar mass (or halo mass) is quite uncertain. Most recently, B05 and T06 concluded that the predicted evolution in the SIS model is considerably stronger than the observed evolution of the rest-frame optical sizes in their stellar mass selected disk samples.

However, the SIS model neglects several important factors that are believed to play a role in determining the size of galactic disks forming in CDM halos. (1) The mass density profiles of CDM halos are not singular isothermal spheres but have a universal form (known as the Navarro-Frenk-White [NFW] profile; Navarro et al. 1997), characterized by the concentration parameter $c_{\text {vir. }}$. The concentration parameter quantifies the density of the halo on small (approximately kiloparsec) scales relative to the virial radius and has an important impact on the structural parameters of the resulting disk. There is a correlation between halo virial mass $M_{\mathrm{vir}}$ and concentration (Navarro et al. 1997), although with a significant scatter (Bullock et al. 2001b, hereafter B01), and this mean halo concentration-mass relation evolves with time, in the sense that halos of a given mass were less concentrated in the past (B01). (2) The self-gravity of the baryonic material may modify the distribution of the dark matter as it becomes condensed in the central part of the halo ("adiabatic contraction"). (3) Disks with low values of $\lambda$ and/or large baryonic-to-dark mass ratios may not have sufficient angular momentum to support a stable disk. These unstable disks may form a bar or a bulge, and might no longer be included in a sample of "disk dominated" galaxies.

There are of course numerous other potential complications in the process of the formation and evolution of galactic disks, which we do not consider here (although we discuss some of them in $\S 5$ ). Here we present the predictions of a model for disk formation that improves on the simple SIS model by incorporating NFW halo profiles, adiabatic contraction, and disk instability. This model is based on the formalism and basic ingredients presented in Blumenthal et al. (1986), Flores et al. (1993), and Mo et al. (1998, hereafter MMW98). Our main result is that the predictions of this improved model are compatible with the rather weak observed evolution of the disk stellar mass-size relation out to $z \sim 1$ reported by B05. We also extend these predictions out to higher redshift, $z \sim 3$, and find acceptable agreement with the results reported by $\mathrm{T} 06$.

We discuss the ingredients of our model in $\S 2$, give a brief summary of the observational data in $\S 3$, present our results in $\S 4$, and discuss our results and conclude in $\S 5$. We assume the following values for the cosmological parameters: matter density $\Omega_{m}=0.3$, baryon density $\Omega_{b}=0.044$, cosmological constant $\Omega_{\Lambda}=0.70$, Hubble parameter $H_{0}=70 \mathrm{~km} \mathrm{~s}^{-1} \mathrm{Mpc}^{-1}$, fluctuation amplitude $\sigma_{8}=0.9$, and a scale-free primordial power spectrum $n_{s}=1$. 


\section{MODEL}

The fundamental hypothesis of our model, following the FallEfstathiou paradigm, is that galactic disks form within massive, extended dark matter halos. The structural properties and multiplicity functions of dark matter halos in a given cosmology can be robustly predicted from $N$-body simulations and analytic fitting formulae. Therefore, the main challenge is to relate the structural properties of dark matter halos to the observable properties of disk galaxies. Making this connection is the goal of our model, which we now briefly present. Note that our approach, and the exposition here, are closely based on the formalism presented in Blumenthal et al. (1986), Flores et al. (1993), and MMW98.

We assume that a fraction $f_{d} \equiv m_{d} / M_{\text {vir }}$ of the halo's virial mass is in the form of baryons that are able to cool and collapse, forming a disk with mass $m_{d}$. We denote the angular momentum of the disk material as $J_{d}$ and assume that it is a fixed fraction of the halo angular momentum $J_{h}$ and that specific angular momentum is conserved when the baryons cool and collapse. We also assume that the disk is thin, in centrifugal balance, and has an exponential surface density profile $\Sigma(r)=\Sigma_{0} \exp \left(-r / r_{d}\right)$. Here $r_{d}$ and $\Sigma_{0}$ are the disk scale length and central surface density and are related to the disk mass through $m_{d}=2 \pi \Sigma_{0} r_{d}^{2}$.

The angular momentum of the disk is

$$
J_{d}=2 \pi \int V_{c}(r) \Sigma(r) r^{2} d r
$$

If we were to assume that the initial dark matter density profile is a singular isothermal sphere and to neglect the self-gravity of the disk, then the rotation velocity $V_{c}(r)$ would be constant and equal to $V_{\text {vir }}$, and the angular momentum of the disk would reduce to

$$
J_{d}=4 \pi \Sigma_{0} V_{c} r_{d}^{3}=2 m_{d} r_{d} V_{\mathrm{vir}}
$$

The spin parameter is defined $\lambda \equiv J_{h}\left|E_{h}\right|^{1 / 2} G^{-1} M_{\mathrm{vir}}^{-5 / 2}$ (Peebles $1969)$, where $E_{h}$ is the total energy of the halo, and we can use this to write

$$
r_{d}=\frac{\lambda G M_{\mathrm{vir}}^{3 / 2}}{2 V_{\mathrm{vir}}\left|E_{h}\right|^{1 / 2}}\left(\frac{J_{d} / m_{d}}{J_{h} / M_{\mathrm{vir}}}\right) .
$$

We now define $f_{j} \equiv\left(J_{d} / m_{d}\right) /\left(J_{h} / M_{\text {vir }}\right)$ to be the ratio of the specific angular momenta of the disk and the halo.

If we assume all particles to be on circular orbits, the total energy of a truncated singular isothermal sphere can be obtained from the virial theorem,

$$
E_{h}=-\frac{G M_{\mathrm{vir}}^{2}}{2 r_{\mathrm{vir}}}=-\frac{M_{\mathrm{vir}} V_{\mathrm{vir}}^{2}}{2} .
$$

If we insert this expression in equation (3), we obtain

$$
r_{d}=\frac{1}{\sqrt{2}} f_{j} \lambda r_{\mathrm{vir}}
$$

If we assume that the spin parameter does not change with cosmic epoch, this leads to the expected scaling $r_{d}(z) \propto r_{\mathrm{vir}}(z)$. We refer to this model as the SIS model, reflecting the assumption that the initial halo profiles are singular isothermal spheres.

A more realistic model would incorporate cosmologically motivated NFW halo profiles (Navarro et al. 1997) and should also include the effect of the self-gravity of the baryons on the predicted properties of the disk. If the gravitational effects of the disk were negligible, then the rotation curve would be the same as that of the unperturbed NFW halo, which rises to a maximum value $V_{\max }$ at a radius $\sim 2 r_{s}$, where $r_{s}$ is the NFW scale radius $r_{s} \equiv r_{\mathrm{vir}} / c_{\mathrm{vir}}$. However, on scales where the mass of baryonic material becomes significant compared with the dark matter, we must account not only for the direct gravitational effects of the baryonic disk material but also for the fact that the dark matter contracts in response to the gravitational force of the baryons as they collapse to form the disk. We compute the effect of this contraction under the assumption that the disk forms gradually enough that the response of the halo is "adiabatic," i.e., that the following "adiabatic invariant" quantity is conserved:

$$
G M_{f}\left(<r_{f}\right) r_{f}=G M_{i}\left(<r_{i}\right) r_{i}
$$

where $M_{i}\left(<r_{i}\right)$ is the initial mass within an initial radius $r_{i}$ (assumed to be given by the NFW profile), and $M_{f}\left(<r_{f}\right)$ is the postcollapse mass within a final radius $r_{f}$. Numerical tests of the validity of the "adiabatic collapse" formalism have shown that it works surprisingly well, even when some of the underlying assumptions (such as spherical symmetry and all particles being on circular orbits) are violated (Jesseit et al. 2002; Gnedin et al. 2004).

The final mass within a radius $r$ is the mass of the exponential disk plus the mass of dark matter within the initial radius $r_{i}$ : $M_{f}(r)=m_{d}(r)+M\left(r_{i}\right)\left(1-f_{d}\right)$. The modified rotation curve can then be written as a sum in quadrature of the contributions from the disk and the (contracted) dark matter halo: $V_{c}^{2}(r)=V_{c, d}^{2}(r)+$ $V_{c, \mathrm{DM}}^{2}(r)$. The rotation curve for the disk is given by the usual expression for a thin exponential disk (see, e.g., Binney \& Tremaine 1987), and the dark matter component of the rotation velocity is given by $V_{c, \mathrm{DM}}^{2}(r)=G\left[M_{f}(r)-m_{d}(r)\right] / r$. Using equation (2) again, and making a change in variable, $u=r / r_{d}$, we can write the expression for the angular momentum of the disk as

$$
J_{d}=m_{d} r_{d} V_{\mathrm{vir}} \int_{0}^{r_{\mathrm{vir}} / r_{d}} e^{-u} u^{2} \frac{V_{c}\left(r_{d} u\right)}{V_{\mathrm{vir}}} d u
$$

and, in direct analogy to equation (5), we obtain

$$
r_{d}=\frac{1}{\sqrt{2}} f_{j} \lambda r_{\mathrm{vir}} f_{c}^{-1 / 2} f_{R}\left(\lambda, c, f_{d}\right)
$$

where

$$
f_{R}\left(\lambda, c, f_{d}\right)=2\left[\int_{0}^{\infty} e^{-u} u^{2} \frac{V_{c}\left(r_{d} u\right)}{V_{\mathrm{vir}}} d u\right]^{-1}
$$

and $f_{c} \equiv E_{\mathrm{NFW}} / E_{\mathrm{SIS}}$ is the total energy for a halo with an NFW profile, relative to that for a singular isothermal sphere with the same mass. Note that in this step we have also used the fact that $r_{\mathrm{vir}} \gg r_{d}$, and that the disk density profile declines exponentially, to set the upper limit of integration to infinity.

For a given set of halo properties $M_{\mathrm{vir}}, c_{\mathrm{vir}}$, and $\lambda$ and disk parameters $f_{d}$ and $f_{j}$, we can solve this system of equations iteratively to obtain $V_{c}(r)$ and $r_{d}{ }^{12}$ We describe how we obtain the halo properties and disk parameters in $\S 2.2$. We refer to the improved model as the NFW model.

By comparing equation (8) with the corresponding expression in the SIS model, equation (5), we can see that they differ by two factors: $f_{c}^{-1 / 2}$, which reflects the difference in energy of a singular

\footnotetext{
12 For a more detailed account of how to go about solving the equations, and analytic fitting functions for $f_{R}$ and $f_{c}$, see MMW98.
} 

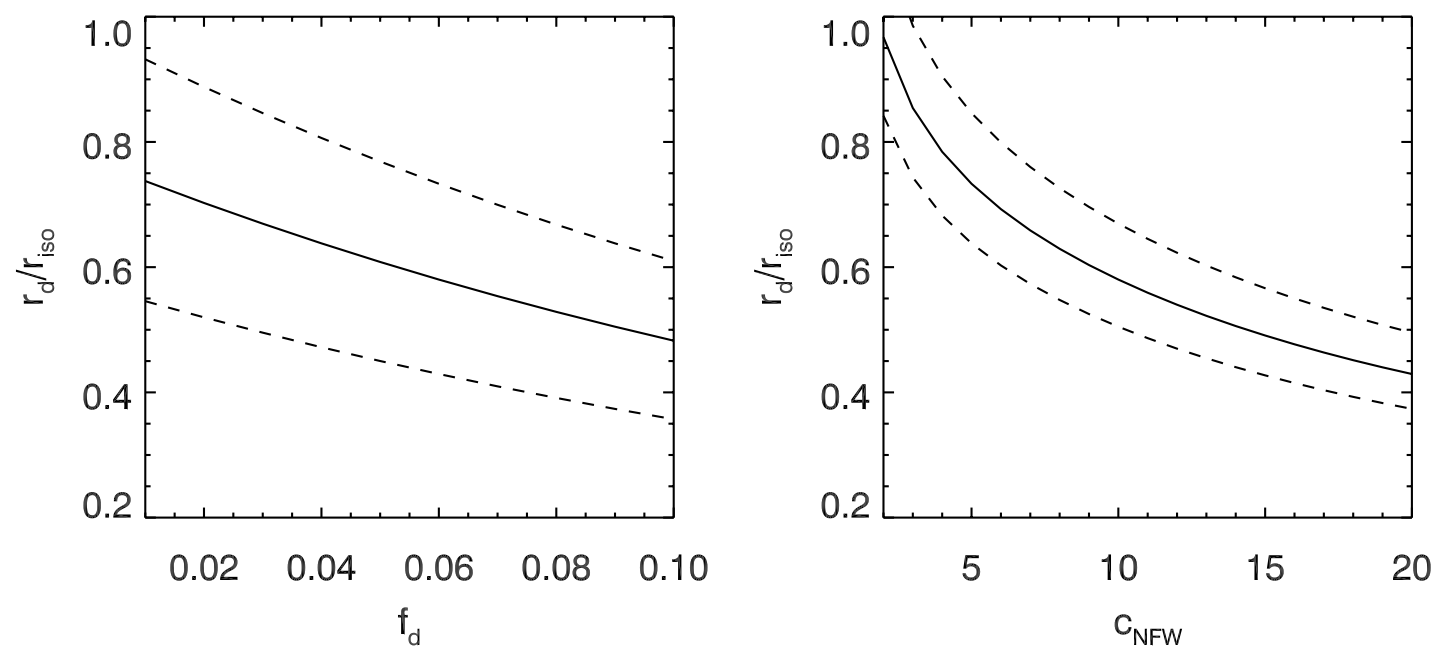

FIG. 1.- Ratio of the disk scale length predicted in the NFW model with adiabatic contraction, to that in the SIS model (neglecting disk self-gravity), as a function of the fraction of baryons in the disk $f_{d}($ left $)$ and the NFW halo concentration $c_{\mathrm{vir}}\left(c_{\mathrm{NFW}}\right.$, right $)$. For all curves, the mean value of the spin parameter $\lambda=0.05$ has been assumed. In the left panel, the solid curves are for $c_{\mathrm{vir}}=10$, while the upper and lower dashed curves are for $c_{\mathrm{vir}}=5$ and 20 , respectively. In the right panel, the solid curve is for $f_{d}=0.06$ and the upper and lower curves are for $f_{d}=0.03$ and 0.09 , respectively.

isothermal sphere versus a NFW profile; and $f_{R}$, which reflects both the NFW density profile and the effect of the adiabatic contraction. The ratio of equations (8) and (5) $\left(r_{d} / r_{\text {iso }}\right)$ is plotted in Figure 1 , as a function of disk fraction $f_{d}$ and of halo concentration $c_{\text {vir }}$. From this plot, we can gain several insights into the behavior of the model. First, we see that the NFW model generally predicts disk sizes that are smaller than the values in the SIS model. For a dark matter halo with $M_{\mathrm{vir}}=10^{12} M_{\odot}$, the present-day virial radius is $r_{\mathrm{vir}} \simeq 260 \mathrm{kpc}$, and so from equation (5), the predicted scale radius would be about $6 \mathrm{kpc}$ for the median value of the spin parameter $\lambda=0.035$. This is almost a factor of 2 larger than one might expect, if such a halo hosts a galaxy similar to the Milky Way or M31. Therefore, there is room for this reduction in size predicted by the new model.

We can also see that smaller values of $f_{d}$ lead to larger values of $r_{d}$. This is because there is less mass and therefore less gravity to contract the halo, leading to a more extended disk. Finally, we see that lower values of concentration $c_{\text {vir }}$ also lead to larger (more extended) disks. Lower values of $c_{\mathrm{vir}}$ imply that there is less mass near the center of the halo relative to the outer parts, again leading to weaker gravitational forces on the kiloparsec scales where the disk is forming, and less contraction of the halo, therefore a more extended disk.

\subsection{Disk Stability}

Because of the rather large range of allowed spin parameters $\lambda$, our model predicts a fairly broad distribution of galaxy sizes at fixed stellar mass. However, it is possible that not all of these disks are gravitationally stable. It is well known from numerical studies that galaxies in which the self-gravity of the baryons dominates may be unstable to the formation of a bar (e.g., Christodoulou et al. 1995). Following MMW98, who base their stability criterion on the study of Efstathiou et al. (1982), we adopt a threshold for instability $\epsilon_{m} \equiv V_{\max } /\left(G m_{d} / r_{d}\right)^{1 / 2}<\epsilon_{m \text {,crit }}$, where $V_{\max }$ is the maximum rotation velocity, which we approximate as the rotation velocity at $\sim 3 r_{d}$. We adopt $\epsilon_{m \text {.crit }}=0.75$ (Syer et al. 1999) and assume that galaxies with $\epsilon_{m}$ less than this critical value may form a significant bar or bulge and be excluded from a disk sample. Because of the uncertainties associated with the fate of these "unstable" disks, however, we present the results of our analysis both including and excluding these objects.

\subsection{Initial Conditions and Model Parameters}

Let us consider a virialized dark matter halo of a given virial mass $\left(M_{\mathrm{vir}}\right)$ at a specific redshift. We require the following information, all as a function of redshift:

1. The number density of these halos.

2. The halo virial radius and virial velocity.

3 . The initial halo mass density profile $\rho(r)$, here characterized by the NFW concentration parameter $c_{\text {vir }}$.

4. The halo specific angular momentum, characterized by the dimensionless spin parameter $\lambda$.

All of this information can be robustly obtained with a high degree of accuracy from modern numerical cosmological $N$-body simulations. We now describe each ingredient in some detail, and how we obtain it.

Halo number density as a function of redshift. - Over a given redshift interval, we select halo masses from the mass function of Sheth \& Tormen (1999) using a Monte Carlo procedure.

Halo virial radius and virial velocity. - It is standard to define dark matter halos as spherical regions within which the average overdensity exceeds a critical value $\Delta_{\text {vir }}$ times the mean background density of the universe. The value of $\Delta_{\text {vir }}$ is computed based on the spherical collapse model. In an Einstein-de Sitter ( $\Omega=1$ ) universe, $\Delta_{\text {vir }}=178$ and by definition the background density $\rho_{b}$ equals the critical density $\rho_{\text {crit. }}$. During the early years of the development of the CDM paradigm, when the Einstein-de Sitter model was widely assumed to be correct, it became common to define halos as overdensities greater than 200 times the critical density (the value 200 was obtained by just rounding off 178). This assumption is still commonly used by many authors today, in spite of the fact that it is no longer well motivated in the now-favored concordance cosmology. We instead use the appropriate value of $\Delta_{\text {vir }}$ for our adopted cosmology, as predicted by the spherical collapse model $\left[\Delta_{\text {vir }}(z=0) \simeq 337\right]$ times the mean background density. This assumption is also commonly used in the literature and was used in the Bullock et al. (2001a, 2001b) papers from which we obtain our halo profile and spin relations. The details of the adaptation of the spherical collapse model to a general cosmology (with cosmological constant) and formulae for and plots of the relations for halo virial radius as a 
function of mass and redshift are given in the appendix of Somerville \& Primack (1999) and are the same relations that we use here. The halo virial velocity is then easily obtained by use of the virial relation, $V_{\mathrm{vir}}=\left(G M_{\mathrm{vir}} / r_{\mathrm{vir}}\right)^{1 / 2}$. Note that because halos are defined with respect to the background density of the universe, average halo densities were higher in the past. This implies that halos of a given virial mass had smaller virial radii, and higher virial velocities, in the past than they do today.

Halo Density Profile.-We assume that initially the halo mass density profiles obey the NFW form (Navarro et al. 1997) and compute the halo concentration $c_{\mathrm{vir}}$ as a function of mass and redshift using the analytic model provided by B01, which is based on the results of numerical simulations. The results of B01 have been confirmed by a number of other studies (Jing 2000; Eke et al. 2001; Wechsler et al. 2002; Macciò et al. 2007).

The basic ingredients of the B01 analytic model for $c_{\mathrm{vir}}(M, z)$ are as follows. They define a "collapse redshift" $z_{c}$ for each halo as $M_{*}\left(z_{c}\right)=F M_{\text {vir }}$, where $\sigma\left[M_{*}(z)\right]=\delta_{c} / D_{\text {lin }}$. Here $F$ is a parameter of the model, which is measured by fitting to $N$-body simulations, $\sigma(M)$ is the $z=0$ linear rms density fluctuation on the comoving scale encompassing a mass $M, \delta_{c} \simeq 1.686$ is the linear theory value of the critical density in the spherical top-hat model, and $D_{\text {lin }}(z)$ is the linear growth factor. Then the concentration is given by $c_{\mathrm{vir}}\left(M_{\mathrm{vir}}, z\right)=K\left(1+z_{c}\right) /(1+z)$, where $K$ is another adjustable parameter of the model. Note that $z_{c}$ is independent of $z$, and therefore $c_{\text {vir }} \propto(1+z)^{-1}$ at fixed mass.

B01 found values of $F=0.01$ and $K=4$ for a $\Lambda$ CDM model with the same values of $\Omega_{m}, \Omega_{\Lambda}$, and $H_{0}$ as our cosmology, but with a higher value of $\sigma_{8}=1$. A recent study by Macciò et al. (2007) based on a larger sample of higher resolution simulations confirms the B01 model but finds $K=3.4$ for a $\Lambda \mathrm{CDM}$ cosmology with $\sigma_{8}=0.9$ (corresponding to a $15 \%$ lower normalization than in B01, but with the same mass dependence). In the model presented here, we adopt the updated Macciò et al. (2007) normalization of the $c_{\mathrm{vir}}\left(M_{\mathrm{vir}}\right)$ relation $(K=3.4)$.

The concentration of halos of a given mass at a given redshift depends on the cosmology and the power spectrum. In cosmologies in which halos assemble their mass early (late), a halo of a given mass has a higher (lower) concentration (Wechsler et al. 2002). We can see from the above formulae that in a cosmology with a modified $D_{\operatorname{lin}}(z)$ (for example, if we changed the value of the cosmological constant, $\left.\Omega_{\Lambda}\right)$, the redshift evolution of $c_{\mathrm{vir}}\left(M_{\mathrm{vir}}\right)$ would be different. Similarly, if we modified the normalization or shape of the power spectrum [which enters through $\sigma(M)$ ], it would alter the normalization and slope of the $c_{\mathrm{vir}}\left(M_{\mathrm{vir}}\right)$ relation. For example, in the cosmology derived from the Wilkinson Microwave Anisotropy Probe (WMAP) three-year results of Spergel et al. $(2007),{ }^{13}$ the $c_{\text {vir }}\left(M_{\text {vir }}\right)$ relation has a normalization about $30 \%$ lower than in our adopted cosmology, but a nearly identical redshift dependence.

Halo Specific Angular Momentum. - N-body simulations have demonstrated that the spin parameter $\lambda$, which characterizes the specific angular momentum of dark matter halos, is uncorrelated with the halo's mass and concentration (Bullock et al. 2001a; Macciò et al. 2007) and does not evolve with redshift. The distribution of $\lambda$ is lognormal, with mean $\bar{\lambda}=0.05$ and width $\sigma_{\lambda}=0.5$ (Bullock et al. 2001a). We therefore assign each halo a value of $\lambda$ by selecting values randomly from this distribution, assuming that it is not correlated with any other halo properties or with redshift.

\footnotetext{
13 The values are $\Omega_{m}=0.24, \Omega_{b}=0.042, \Omega_{\Lambda}=0.76, H_{0}=73 \mathrm{~km} \mathrm{~s}^{-1} \mathrm{Mpc}^{-1}$, $\sigma_{8}=0.74$, and $n_{s}=0.95$.
}

\subsection{Predicting Observable Disk Properties}

We assume that each halo with a virial velocity below $350 \mathrm{~km} \mathrm{~s}^{-1}$ hosts one disk galaxy at its center. Once the halo properties are specified as described above (based on dissipationless $N$-body simulations), there are only two free parameters in our model: the fraction of mass in the form of baryons in the disk, $f_{d}$, and the fraction of the specific angular momentum of the DM halo captured by the disk, $f_{j}$. Throughout this work, we assume $f_{j}=1$. Although there are physical reasons to think that $f_{j}$ may not be exactly equal to unity, note that in order to change our main conclusions, $f_{j}$ would have to be a strong function of redshift. There is no obvious physical reason to expect this to be the case.

Since $f_{d}$ is now the only free parameter, we can fix its value by requiring that we reproduce the observed scale length of a galaxy of a given virial mass scale. For example, adopting $f_{d}=0.06$ produces a galaxy with disk mass $m_{d}=6 \times 10^{10} M_{\odot}$ and scale length $r_{d}=3.6 \mathrm{kpc}$ in a halo of mass $M_{\mathrm{vir}}=10^{12} M_{\odot}$, similar to the Milky Way and in good agreement with the median value for the scale length of disks in our SDSS sample at this stellar mass. However, if we assume a constant value of $f_{d}=0.06$ for all halo masses, we find that the slope of the stellar mass versus disk scale length relation is too steep; i.e., the sizes of smaller mass disks are too small, and those of larger mass disks too large, compared with the observed relation. This has also been noted before by other authors working with similar models (e.g., S03; Dutton et al. 2007). We can solve this problem by assuming that the disk baryon fraction varies with halo mass, in the sense that lower mass halos form disks with lower baryon fractions. This is in fact what is found in physically motivated models of galaxy formation, as supernova feedback can more easily heat and eject gas from lowmass halos (Natarajan 1999; Dekel \& Silk 1986). We therefore adopt a simple functional form for $f_{d}$ as a function of halo mass, $f_{d}=f_{0} /\left[1.0+\left(M_{\mathrm{vir}} / M_{c}\right)^{\alpha}\right]$, following S03, and find good agreement with the observed stellar mass versus disk scale length relation with parameter values $f_{0}=0.13, M_{c}=1.0 \times 10^{12} M_{\odot}$, and $\alpha=-0.67$. Note that we do not discriminate between stellar mass and the mass in cold gas. For the relatively massive disks that we focus on, the cold gas fraction should be fairly low.

\section{SUMMARY OF OBSERVATIONAL DATA}

For the main part of our analysis, we use the same sample of disk-dominated galaxies that was used for the analysis of B05. We give a brief summary of that sample here, and refer to B05 for details. For our local $z \sim 0$ sample, we use the New York University Value-added Galaxy Catalog (VAGC; Blanton et al. 2005), based on the second data release of the SDSS (DR2). The VAGC catalog was used to obtain Sérsic parameters, $r$-band half-light radii, and magnitudes. We then construct a sample of diskdominated galaxies by requiring Sérsic parameter $n<2.5$. We convert the $r$-band half-light radii to rest-frame $V$-band radii using the conversion derived in $\mathrm{B} 05, R_{e}(V)=1.011 R_{e}(r)$. We compute stellar masses from the ( $k$-corrected) $g$ - and $r$-band photometry, using the prescription of Bell et al. (2003), which relies on a conversion between $g-r$ color and average stellar mass-to-light ratio. We assume a normalization for this relation consistent with a Kroupa (2001) initial mass function (IMF).

Our high-redshift disk sample comes from the GEMS survey ( Rix et al. 2004; Caldwell et al. 2005), which consists of $V_{606}$ and $z_{850}$ imaging over an area of $\sim 900 \operatorname{arcmin}^{2}$ with the ACS on $H S T$. The $5 \sigma$ point-source detection limit is $28.3 \mathrm{mag}$ in the $V_{606}$ band and $27.1 \mathrm{mag}$ in $z_{850}$. The GEMS object catalog is based on the $z_{850}$ image; for details see Caldwell et al. (2005). High-accuracy photometric redshift estimates $\left[\sigma_{z} /(z+1) \sim 0.02\right]$ are obtained 
from the ground-based COMBO-17 survey (Wolf et al. 2004). The $R$-band selection limit of COMBO-17 $\left(m_{R} \sim 24\right)$ limits the range of the sample to redshifts $z \lesssim 1$. The 17-band photometry of COMBO has also been used to obtain stellar mass estimates (Borch et al. 2006), assuming a Kroupa IMF. The GEMS main sample consists of almost 8000 galaxies with COMBO counterparts and redshifts. We fit each galaxy with a Sérsic profile and select a disk-dominated sample with good quality fits, Sérsic $n<2.5$, and extended light profiles. This sample contains 5664 objects. Typical uncertainties are $\sim 35 \%$ in $r_{e}$ and $\sim 0.2 \mathrm{mag}$ in $m_{z}$ (Häussler et al. 2007). The apparent half-light sizes measured in the observed $z_{850}$ band are converted to rest-frame $V$ band using an average color gradient correction based on a local sample of disk galaxies (see B05). These corrections are small $( \pm 3 \%)$ over the entire redshift range of our sample (the observed $z_{850}$ band samples the rest-frame $V$ band at $z \sim 0.5$ ). Where disk scale radii are quoted in this work, we have obtained them by simply assuming that the measured half-light radius and the disk scale radius are related by the standard expression for a pure exponential disk, $r_{d}=r_{e} / 1.68$.

In order to estimate the completeness of the combined GEMS + COMBO disk sample, we have performed extensive simulations (Häussler et al. 2007; Rix et al. 2004). Artificial disks were inserted into blank sky, and the source detection and fitting software was run on this image. Poor fits are excluded in the same manner as for the real galaxy images. We can then calculate the success rate for detecting and obtaining a good fit for the artificial galaxies, as a function of apparent effective radius and apparent magnitude. We multiply this GEMS completeness factor by the (redshift, magnitude, and color dependent) probability that the galaxy would be detected and successfully assigned a redshift in the COMBO survey. Based on these estimates, B05 argue that GEMS is not surface brightness limited even in the highest redshift bin and that the combined GEMS + COMBO sample is complete down to stellar masses of $10^{10} M_{\odot}$. As in B05, we limit our analysis to galaxies with stellar mass greater than this value, and we weigh galaxies by the inverse completeness factor in computing distributions and means. To avoid using galaxies with very large weights, we exclude objects with a completeness factor smaller than 0.5. B05 have shown that the average sizes and surface densities are insensitive to the choice of this limiting completeness factor at our adopted stellar mass limit.

\section{RESULTS}

Using the models outlined above, we construct a mock catalog for a low-redshift slice $(0.001<z<0.2)$ representative of the SDSS sample, as well as a light cone from $0.1<z<1.1$ with approximately 10 times the area of GEMS $\left(10 \times 900 \operatorname{arcmin}^{2}\right)$, which we divide into five bins with roughly equal comoving volume $(0.1<z<0.56,0.56<z<0.74,0.74<z<0.87,0.87<$ $z<1.0$, and $1.0<z<1.1$ ). Following MMW98, we define "stable disks" as those with stability parameter $\epsilon_{m}$ above a critical value and adopt $\epsilon_{m, \text { crit }}=0.75$ (Syer et al. 1999). The stellar mass versus disk scale length relations for the six redshift bins from $z \sim 0$ to 1 are shown in Figure 2, for the mock catalogs on the left and the observed SDSS and GEMS disk samples on the right. ${ }^{14}$ The observational samples are corrected for completeness as described in $\S 3$.

\footnotetext{
${ }^{14}$ Note that, strictly speaking, the models predict the scale length of the baryonic mass in the disk, while the observed scale lengths are for the rest-frame $V$-band light. We do not attempt to correct for the known $\sim 20 \%$ difference between these two quantities, since we are mainly interested in the redshift evolution. We do note here, however, that time-evolving color gradients could therefore change our results.
}

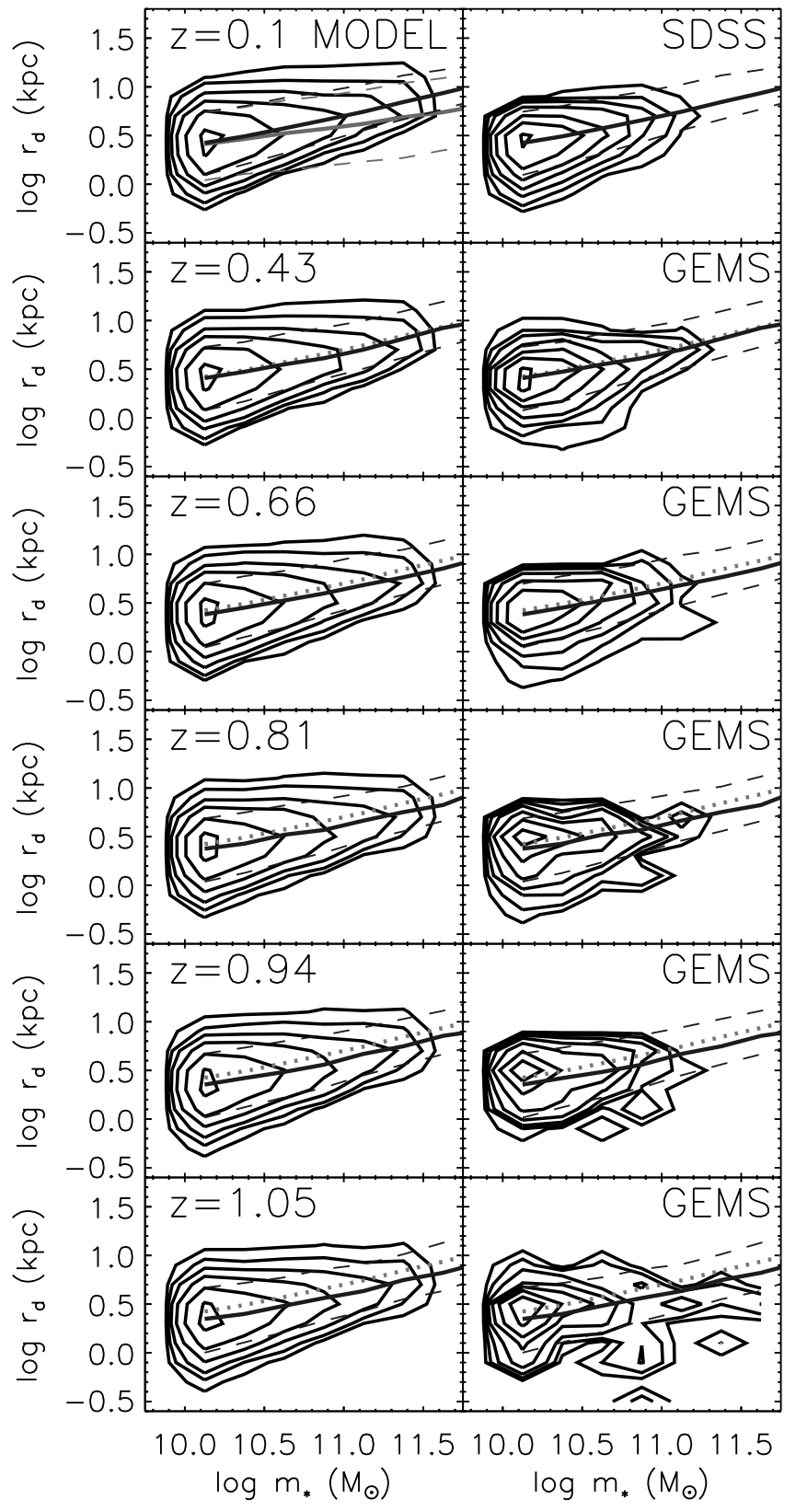

Fig. 2.- Relationship between stellar mass and disk scale length for a "local" sample $(0.001<z<0.2)$ and in five redshift bins of approximately equal comoving volume from $z=0.1$ to 1.1 . In the left panels, contours show the NFW model predictions for stable disks. In the right panels, contours show the completeness-corrected distributions for the SDSS and GEMS samples for the same redshift bins. The left and right panels are normalized to the same total number density in each redshift bin. The solid and dashed lines show the median and 10th and 90th percentiles, respectively, for the stable model disks in both columns of panels (models and data). The solid and dashed lines in the left $z=0.1$ panel (top left) show the median and 10th and 90th percentiles for all disks, without any stability criterion applied. The dotted lines, repeated in each panel, show the $z=0.1$ medians and 10th and 90th percentile lines for the stable model disks. [See the electronic edition of the Journal for a color version of this figure.]

\subsection{The Size-Mass Relation and Its Evolution to $z \sim 1$}

In the left panels of Figure 2, the contours show the results for stable disks only. To illustrate the impact of excluding the unstable disks, the median and 10th and 90th percentiles in size as a function of stellar mass for all disks, without any stability criterion applied, are shown in the $z=0.1$ panel (top left). The relative impact of applying the stability criterion on the size-mass 


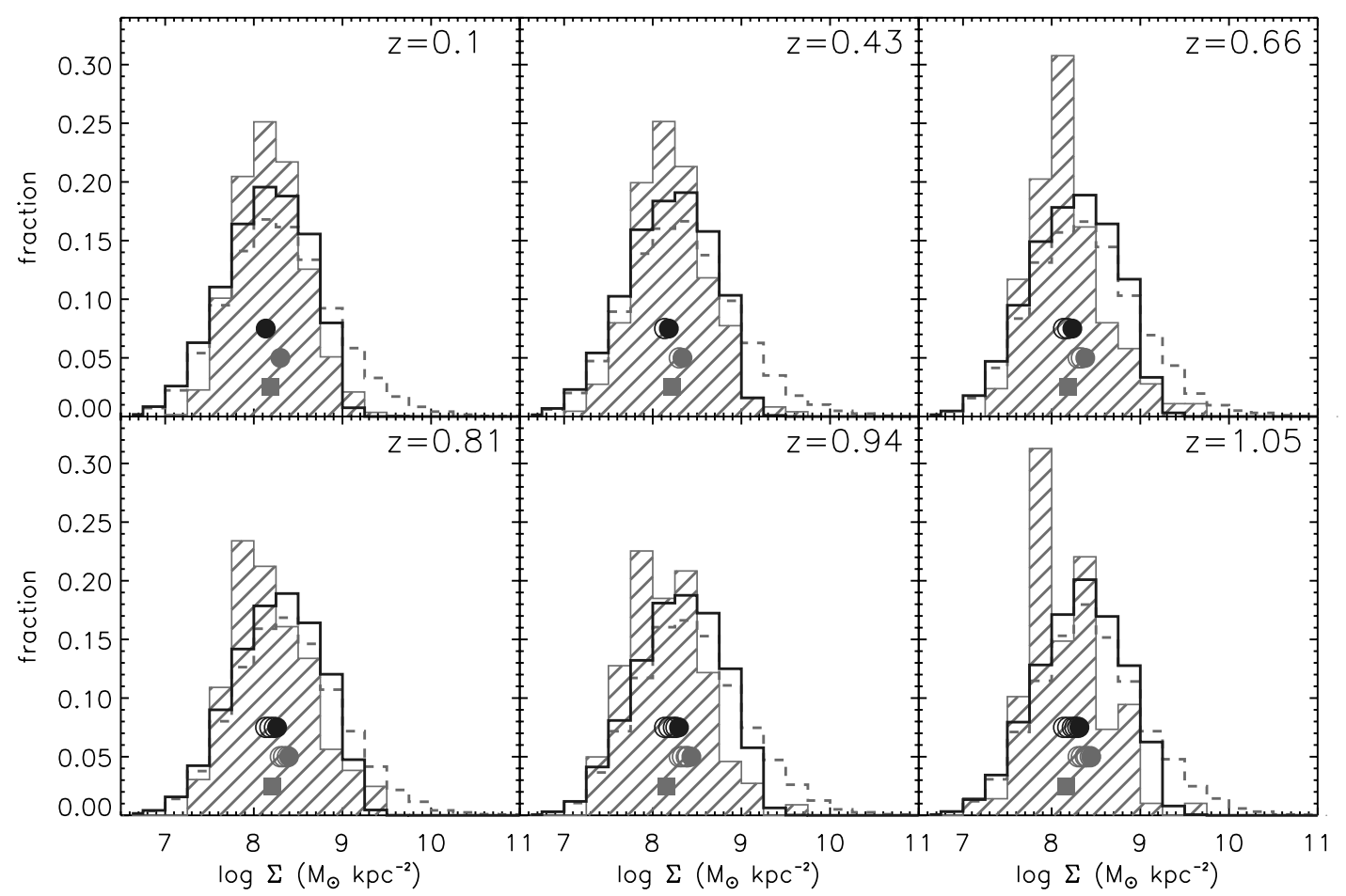

FIG. 3.-Distribution of stellar surface densities for disks with stellar mass $10^{10} M_{\odot}<m_{*}<10^{11} M_{\odot}$, for a "local" sample $(0.001<z<0.2)$, and in five redshift bins of approximately equal comoving volume from $z=0.1$ to 1.1 . Hatched histograms show the completeness-corrected distributions of SDSS and GEMS disks, selected via Sérsic fits to their radial light profiles $(n<2.5)$. Filled squares show the mean value of $\log \Sigma_{*}$ derived from these observations. Dashed lines show the distributions for all model disks, and solid lines show the results for "stable" model disks only (see text). The filled circles indicate the mean of the model distribution for the current redshift bin, and the open circles show the means from all lower redshift bins. The black filled circles are for stable disks, and the gray filled circles are for all disks. [See the electronic edition of the Journal for a color version of this figure.]

relation and its scatter is similar in all the redshift bins considered here, so for clarity we show only the median relation for stable disks in the other panels. It is clear from the definition of the stability parameter $\epsilon_{m}$ that at a given halo mass, disks with larger values of $f_{d}$ are more likely to be unstable, and also at a given stellar mass, more compact disks (low $r_{d}$ ) are more likely to be unstable. Therefore, more massive galaxies are more likely to be unstable (recall that we assumed that $f_{d}$ increases with increasing halo mass), and so excluding the unstable (compact) disks results in a steepening of the slope in the $m_{*}-r_{d}$ relation at $m_{*} \simeq(2-3) \times$ $10^{10} M_{\odot}$. Excluding the unstable disks also significantly reduces the scatter in disk size at fixed stellar mass for the massive disks. The fraction of disks deemed stable by our criterion is nearly constant over the redshift range considered here and ranges from $95 \%$ at $z \sim 0.1$ to $97 \%$ at $z \sim 1$.

Figure 2 shows that the NFW model can reproduce both the observed slope of the size-mass relation and the scatter in size at a given stellar mass fairly well at $z \sim 0$. Our results agree with those of S03 and other investigations. The median and 10th and 90th percentiles in disk scale length as a function of stellar mass for the stable model disks from the low-redshift bin are repeated in every panel, and against these contours we can immediately see that the model predicts that the average size of disks at fixed stellar mass has increased by about $15 \%-20 \%$ since $z \sim 1$. The GEMS sample is consistent with no evolution in size at fixed stellar mass. Note that we have normalized the model and data histograms to the same total number density in each redshift bin.

An important side issue is that we could see in Figure 2 that the model produces too many massive disks $\left(m_{*}>10^{11} M_{\odot}\right)$, especially at high redshift, compared with the observational samples. This is not surprising, since we have assumed that every dark matter halo contains a single disk galaxy, which is clearly not realistic, and we know that more massive halos (which produce the massive disks) are more likely to instead host an early-type galaxy. Therefore, in order to avoid any bias from the unrealistically massive model galaxies, in Figures 3 and 4 we show these distributions for galaxies with stellar masses in the range $10^{10} M_{\odot}<m_{*}<10^{11} M_{\odot}$.

Figure 3 shows the distribution of stellar surface densities $\Sigma_{*}$ for the same six redshift bins from $z \sim 0.1$ to $\sim 1$. We define the stellar surface density as $\log \Sigma_{*}=\log m_{*}-2 \log r_{e}-\log (2 \pi)$, where $r_{e}=1.68 r_{d}$. From this figure, we can readily see that if we had not excluded unstable disks, the model would have predicted a broader distribution of surface densities than is seen in the data, with a highly skewed tail to very high densities that are not observed in the disk samples. With unstable disks excluded, the width of this distribution is reasonably consistent with the data at all redshifts, although there are hints of some interesting discrepancies; namely, the observational distributions appear perhaps a bit narrower and a bit more skewed than the model predictions.

Figure 4 shows the evolution of the average value of $\log \Sigma_{*}$ for the same stellar mass range. As seen in Figure 3, the model predicts significant, but fairly mild, evolution in $\log \Sigma_{*}$ over the redshift range considered (about $0.2 \mathrm{dex}$, or about a factor of 1.5; consistent with $\left.\Sigma_{*} \propto r_{e}^{-2}\right)$. We also show the SIS scalings both for $r_{200}$ and for $r_{\text {vir }}$ (see $\S 2$ ). ${ }^{15}$ Both of these predict much more dramatic redshift evolution than is observed: as found in B05, and as seen here, the results from the GEMS analysis are consistent

\footnotetext{
${ }^{15}$ Note that the evolution is somewhat more rapid when the definition $r_{\text {vir }}$ is used, because the virial overdensity used to define the halo, $\Delta_{\text {vir }}$, evolves with redshift while with the $r_{200}$ definition it remains constant. Because $\Delta_{\text {vir }}$ is larger at higher redshift, the halos are smaller in radius.
} 


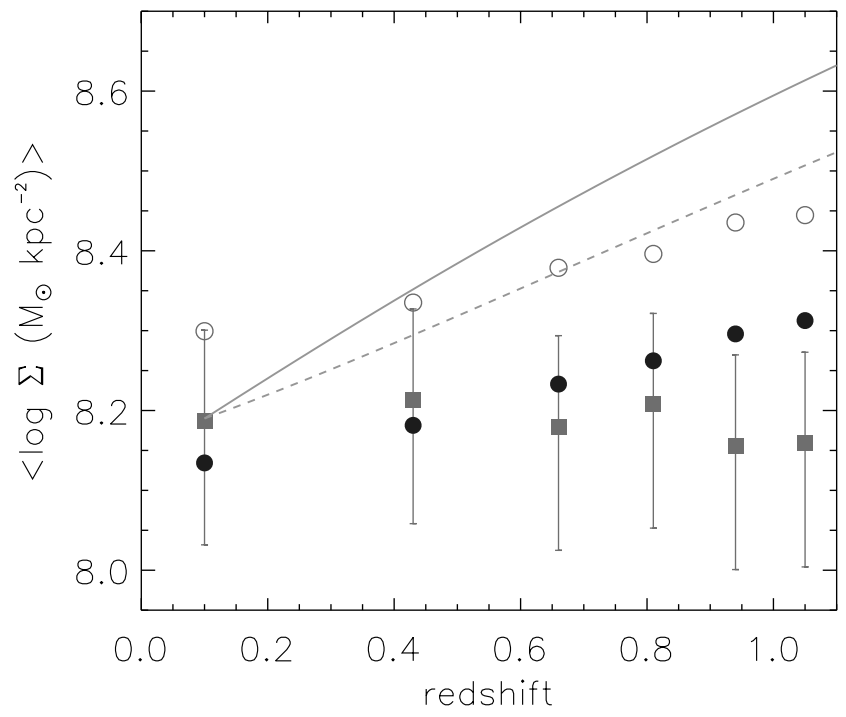

FIG. 4.- Redshift evolution of the mean stellar surface density. Filled squares show the mean of $\log \Sigma_{*}$ for SDSS and GEMS disks with $10^{10} M_{\odot}<m_{*}<$ $10^{11} M_{\odot}$. Open circles show the results for all NFW model disks in this mass range, and filled circles show the results for stable model disks only. The dashed curve shows the evolution in $\Sigma_{*}$ that we would expect if disk size scaled like $r_{200}$, and the solid curve shows the evolution for disk sizes that scale like $r_{\text {vir }}$ (as in the SIS model; see text). The SIS model predicts that disk surface densities were considerably higher at $z \sim 1$, in conflict with the data. [See the electronic edition of the Journal for a color version of this figure.]

with no evolution in the average value of $\Sigma_{*}$ over the redshift range $0.1 \lesssim z \lesssim 1.1$.

B05 found that the $2 \sigma$ error bars on the average values of $\log \Sigma$ obtained from bootstrap resampling are $\sim \pm 0.04-0.1 \mathrm{dex}$, but this certainly underestimates the possible systematic errors. For example, the stellar mass estimates may be systematically incorrect if galaxies have more bursty star formation histories at high redshift, or the size estimates could be systematically biased by the increasingly irregular morphologies of high-redshift disks (our fitting simulations assume perfectly smooth galaxies). We estimate the overall systematic uncertainty in size at fixed stellar mass to be $\sim 30 \%$, and show representative error bars reflecting this in Figure 4. The prediction of the NFW model is a significant improvement over the SIS scaling, and it is in acceptable agreement with the GEMS data to $z \sim 1$ within these estimated uncertainties.

The surface density of disks is important because nearly all semianalytic galaxy formation models assume that the efficiency of star formation is determined by the disk surface density through the Kennicutt law, $\dot{\Sigma}_{*} \propto \Sigma_{\text {gas }}^{N}$, above a critical surface density $\Sigma_{\text {crit }}$. Predicting disk surface densities correctly is therefore very important for self-consistent models of galaxy formation. Note, however, that many semianalytic models (e.g., Somerville \& Primack 1999; Kauffmann et al. 1999; Croton et al. 2006) effectively assume that the disk surface density scales according to the SIS prediction. This will clearly lead to fairly serious inaccuracies in the predictions of the evolution of the star formation rates in galaxies in these models.

\subsection{Evolution to Higher Redshift}

We now briefly explore how the models fare in comparison with the more limited data available at higher redshift. Figure 5 shows the average size of disk galaxies with stellar mass greater than $3 \times 10^{10} M_{\odot}$ predicted by the model, out to $z \sim 3$, compared with the combined results from SDSS, GEMS, and FIRES, presented by T06. Each set of points is normalized relative to the

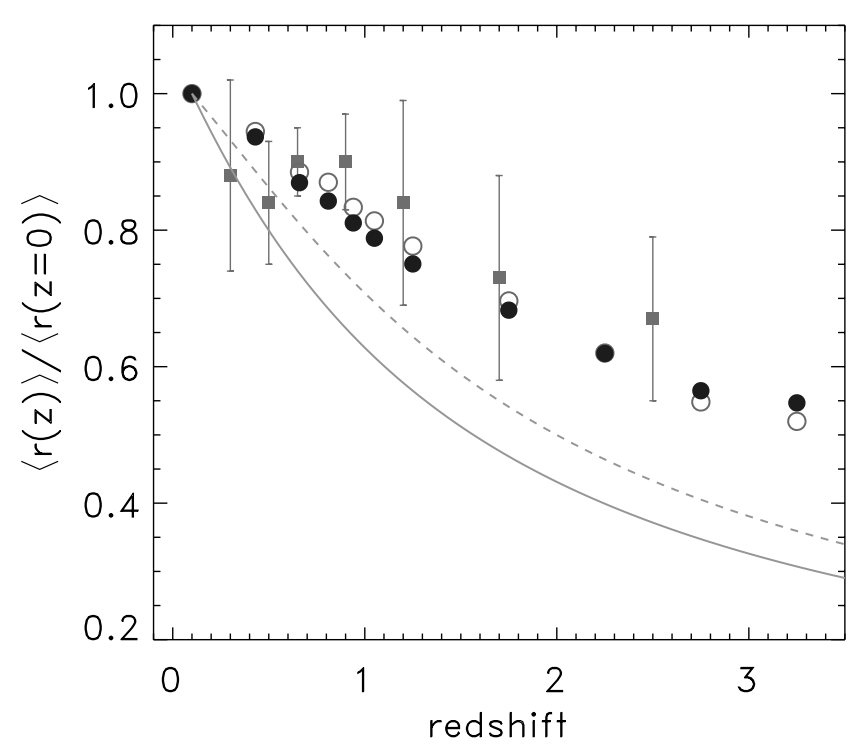

FIG. 5.- Redshift evolution of the average size of disks with stellar masses greater than $3 \times 10^{10} M_{\odot}$, relative to the average size of disks at $z=0.1$. Filled squares with error bars show the observational estimates from T06, obtained by combining the SDSS, GEMS, and FIRES data sets. Open circles show the NFW model predictions for all disks. Filled circles show the NFW model predictions for stable disks only. The dashed and solid curves show the scaling of $r_{200}$ and $r_{\text {vir }}$, respectively, for dark matter halos of fixed mass, as in the SIS model. The NFW model predicts more gradual size evolution, in better agreement with the observations than the SIS model. [See the electronic edition of the Journal for a color version of this figure.]

average scale length of that sample at $z=0.1$. Results are shown for all disks, and for "stable" disks only. Note that the absolute average disk scale lengths for the "stable" disks are always larger than those for the total set of disks, with no stability criterion applied; however, the redshift evolution of the stable disk sample is slightly steeper than that of the overall sample out to $z \sim 2$. This is why the stable disk sizes, when normalized relative to the average size of stable disks at $z=0$, are a little bit lower than the total disk sample out to $z \sim 2$. We again also show the scaling for halo virial radius at fixed halo mass, which would predict much more rapid size evolution than is observed. As we have already seen, the improved model predicts fairly mild evolution in the average disk sizes out to $z \sim 1$, in quite good agreement with the GEMS data. At higher redshifts of $z \sim 2-3$, our model predicts that disks should be about $60 \%$ as large as they are today at a given stellar mass. This represents somewhat more evolution than the observational results of T06 indicate, but is within the quoted error bars.

\subsection{Evolution of the Tully-Fisher Relation}

Our models also simultaneously predict the rotation curves of the disks. It has been shown in previous work that models similar to that presented here can reproduce the detailed rotation curves for the Milky Way and M31 (Klypin et al. 2002) and the TullyFisher relation (circular velocity at some fiducial radius vs. luminosity, or vs. stellar or baryonic mass) at redshift zero (MMW98; Dutton et al. 2007; Gnedin et al. 2006). A detailed comparison with observations of rotation velocities and the Tully-Fisher relation is beyond the scope of this paper, but we briefly present a prediction for the evolution of the Tully-Fisher zero point (here expressed as the average rotation velocity at $\sim 3$ disk scale lengths, for disk galaxies of a fixed stellar mass, $\left.m_{*} \sim 6 \times 10^{10} M_{\odot}\right)$ in Figure 6 . We find that the disk rotation velocity $V_{\text {disk }} \equiv V_{c}(r=$ $3 r_{s}$ ) at fixed disk mass evolves much more gradually than the halo 


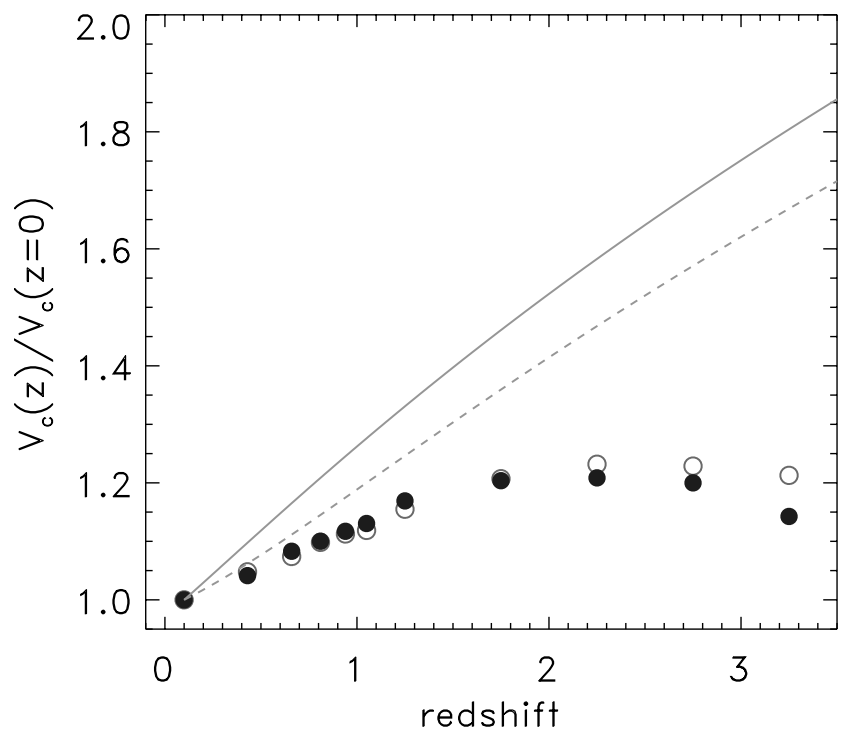

FIG. 6.- Redshift evolution of the circular velocity of disks with stellar mass $(5-7) \times 10^{10} M_{\odot}$, relative to the average circular velocity of such disks at $z=0.1$. Open circles show the model predictions for all disks. Filled circles show the model predictions for stable disks only. The dashed and solid curves show the scaling of $V_{200}$ and $V_{\text {vir }}$, respectively, for dark matter halos of fixed mass, as in the SIS model. The NFW model predicts more gradual evolution in rotation velocity, in qualitative agreement with Tully-Fisher observations to $z \sim 1$ (see text). [See the electronic edition of the Journal for a color version of this figure.]

virial velocity $V_{\text {vir }}$ at fixed halo mass. In fact, our model predicts that $V_{\text {disk }}$ should actually remain nearly constant from $z \sim 1.5$ to 3 . Observational constraints on the time evolution of the Tully-Fisher relation, particularly in terms of stellar or baryonic mass, remain uncertain, but Conselice et al. (2005) found no evolution in the $K$ band or stellar mass Tully-Fisher to $z \sim 1$, and Kassin et al. (2007) also find no evolution to $z \sim 1$ in a redefined version of the stellar mass Tully-Fisher relation. ${ }^{16}$

\section{DISCUSSION AND CONCLUSIONS}

We have shown that a CDM-based model of disk formation produces good agreement with the observed weak redshift evolution of the disk size-stellar mass relation from GEMS out to $z \sim 1$. This result is in contrast to the considerably stronger evolution implied by the assumption often used in the literature, that disk sizes simply scale in proportion to the dark matter halo virial radii. Similarly, we find much weaker evolution in the disk rotation velocity at a given disk mass than we would expect if $V_{\text {disk }}$ scaled in proportion to the halo virial velocity $V_{\text {vir. }}$.

The more gradual evolution in the "improved" model results primarily from the use of realistic NFW halo profiles and from the incorporation of the redshift evolution of the halo concentrationmass relation predicted by $N$-body simulations. Recall that in our model, at fixed halo mass, there are two halo properties and two disk parameters that determine the structural properties of the disk: halo concentration $c_{\mathrm{vir}}$, halo spin $\lambda$, the baryon fraction of the disk $f_{d}$, and the fraction of specific angular momentum captured by the disk material $f_{j}$. We have assumed that the distribution of spin parameters $\lambda$ for the overall population of dark matter halos does not change with time, as demonstrated by $N$-body simulations. We have also assumed that $f_{d}$ is a fixed function of halo mass that does not depend on redshift (although in reality this may not be true),

\footnotetext{
16 Instead of using the usual rotation velocity, they define a new velocity variable which includes contributions from random motions as well as rotation.
}

and we have assumed a fixed value of $f_{j}=1$. Therefore, there is only one ingredient left that can possibly be responsible for changing the predictions for the redshift dependence of disk sizes at fixed mass: the halo concentration versus mass relation.

As we have discussed, simulations have shown that the halo concentration versus mass relation depends on redshift: the halo concentration at fixed mass scales as $c_{\text {vir }} \propto(1+z)^{-1}$ (B01). Therefore, a halo of a given mass is less concentrated at high redshift. This apparent evolution, however, is really a consequence of the way that halos are assembled in a CDM universe. Studies of the mass accretion history of halos in simulations have shown that they have two basic phases of growth: an early, rapid phase, in which the central density is set, and a second phase of more gradual accretion (Wechsler et al. 2002). The mass within the characteristic scale radius $r_{s}$ is assembled during the early, rapid accretion phase. Afterward, $r_{s}$ stays nearly constant, while $r_{\text {vir }}$ increases due to smooth accretion of mass, leading to a formal decrease in $c_{\text {vir }} \equiv r_{\text {vir }} / r_{s}$.

In $\oint 2$ we demonstrated that less concentrated halos produce larger disks because of the lower mass and weaker gravitational forces in the central parts of the halo, where the disk forms. Thus, the collapse of baryons produces less contraction of the dark matter profile, and a more extended disk. The trend toward lower concentrations at earlier times therefore counteracts the decreasing virial radii. Out to about $z \sim 1$, these competing effects nearly cancel out, leading to the weak evolution in the size-mass relation that we have shown.

Note that we have repeated our calculations using the WMAP three-year cosmological parameters derived by Spergel et al. (2007). Because of the reduced small scale power in this cosmology, halos form somewhat later and therefore have lower concentrations for their mass at the present day. However, once we renormalize our model (by making a minor adjustment to the normalization of the disk fraction relation $f_{d}$ ) to again reproduce the size-mass relation of present-day disks, we find that the redshift evolution of disk sizes and circular velocities is nearly unchanged. This is not surprising, as the redshift evolution of the halo concentration versus mass relation is nearly identical to the one we originally adopted.

We see a hint that the evolution predicted by these models is still a bit stronger than that indicated by the data. This could be a sign that one of the other assumptions in our simple model is incorrect. For example, if the disk baryon fraction $f_{d}$ at a given halo mass decreases with increasing redshift, this would lead to shallower evolution and relatively larger disks at high redshift. Because we have measured the disk sizes in the rest-frame $V$ band, evolving color gradients could also mask evolution in the true size of the stellar disk. Alternatively, systematic biases in our stellar mass and size estimates could be impacting the observational estimates.

We also see an increasing level of discrepancy at higher redshifts, $z \gtrsim 1.5$. This could be a hint that an entirely different mechanism could be responsible for setting the sizes of disks at very high redshift. Mergers between gas-rich disks could result in a new, more spatially extended disk (Kazantzidis et al. 2005; Springel \& Hernquist 2005; Robertson et al. 2006). The same scenario could also help to explain the kinematics of damped Ly $\alpha$ systems, which are difficult to reconcile with the standard Fall-Efstathiou picture of disk formation (Maller et al. 2001).

We also presented a prediction for the redshift evolution of the average rotation velocity at $\sim 3 r_{d}$ (close to the maximum of the rotation curve) for disks of fixed stellar mass $m_{*} \sim 6 \times 10^{10} M_{\odot}$ (i.e., the zero point of the Tully-Fisher relation). We found a result similar to that found for the disk sizes: $V_{\text {disk }}$ decreases with 
time, but much more slowly than we would expect if we assumed that $V_{\text {disk }} \propto V_{\text {vir. }}$. The physical reason for this is the same as the one we have discussed in relation to the weak size evolution: halos have lower concentrations at high redshift, and thus less mass near the center and so lower rotation speeds at the radii where rotation velocities are measured (near the maximum value of the rotation curve, $V_{\max }$ ). Thus, $V_{\max }$ even for pure DM NFW halos, without accounting for baryons, evolves more slowly than $V_{\text {vir }}(\mathrm{B} 01)$. In addition, in our model, lower concentrations lead to less contraction and more extended disks. This leads to flatter, less "peaky" rotation curves and lower values of $V_{\text {disk }}$ relative to a more concentrated halo (MMW98; Klypin et al. 2002).

About the same fraction of disks are classified as unstable according to the condition we adopted $\left(\epsilon_{m}<0.75\right)$ over the whole redshift interval $0 \leq z \leq 3$, and the exclusion of unstable disks from the sample changes the average size by nearly the same amount over this interval as well. Therefore, as implemented here, disk stability does not play a significant role in determining the relative time evolution of the stellar mass-size relation, although it is important for reproducing the correct distribution of disk stellar surface densities. At very high redshifts, $z \gtrsim 2.5$, our model predicts that the number of unstable disks starts to increase. However, we do not even know that thin, rotationally supported disks exist at such high redshifts, so we do not know how seriously to take this prediction.

While the model presented here represents a significant improvement with respect to the overly simplified $\lambda r_{\text {vir }}$ scaling commonly used in the literature, it still neglects many important aspects of disk formation in a hierarchical universe, in particular the impact of mergers. We have also ignored the possible presence of spheroids and cold gas in our disk galaxies. In addition, the fraction of baryons in the disk component as a function of halo mass $\left(f_{d}\right)$, here assumed to be a simple deterministic function, almost certainly has a large scatter and may change systematically with time. We intend to investigate the predictions of more detailed models, set within hierarchical merger trees and including a full treatment of cooling, star formation, feedback, etc., in a future work, in which we will also explore the redshift evolution of the disk size function (R. Somerville et al., in preparation).

This work is based on observations taken with the NASA/ESA Hubble Space Telescope, which is operated by the Association of Universities for Research in Astronomy, Inc. (AURA), under NASA contract NAS5-26555. Support for the GEMS project was provided by NASA through grant GO-9500 from the Space Telescope Science Institute, which is operated by AURA for NASA under contract NAS5-26555. Support for this work also came from HST archival grant AR-10290. S. F. S. acknowledges financial support provided through the European Community's Human Potential Program under contract HPRN-CT-2002-00305, Euro3D RTN. E. F. B. is supported by the DFG's Emmy Noether Program. C. W. was supported by a PPARC Advanced Fellowship. S. J. acknowledges support from NASA under LTSA grant NAG5-13063 issued through the Office of Space Science. D. H. M. acknowledges support from NASA under LTSA grant NAG513102 issued through the Office of Space Science. C. H. is supported by a CITA National Fellowship. K. J. acknowledges support by the German DFG under grant SCHI 536/3-1.

Funding for the Sloan Digital Sky Survey (SDSS) has been provided by the Alfred P. Sloan Foundation, the Participating Institutions, the National Aeronautics and Space Administration, the National Science Foundation, the US Department of Energy, the Japanese Monbukagakusho, and the Max Planck Society. The SDSS Web site is http://www.sdss.org. The SDSS is managed by the Astrophysical Research Consortium (ARC) for the Participating Institutions. The Participating Institutions are the University of Chicago, Fermilab, the Institute for Advanced Study, the Japan Participation Group, the Johns Hopkins University, Los Alamos National Laboratory, the Max-Planck-Institute for Astronomy (MPIA), the Max-Planck-Institute for Astrophysics (MPA), New Mexico State University, University of Pittsburgh, Princeton University, the United States Naval Observatory, and the University of Washington.
Avila-Reese, V., Firmani, C., \& Hernández, X. 1998, ApJ, 505, 37 Barden, M., et al. 2005, ApJ, 635, 959 (B05)

Bell, E. F., McIntosh, D. H., Katz, N., \& Weinberg, M. D. 2003, ApJS, 149, 289

Binney, J., \& Tremaine, S. 1987, Galactic Dynamics (Princeton: Princeton Univ. Press)

Blanton, M. R., et al. 2005, AJ, 129, 2562

Blumenthal, G., Faber, S., Flores, R., \& Primack, J. 1986, ApJ, 301, 27

Borch, A., et al. 2006, A\&A, 453, 869

Bullock, J. S., Dekel, A., Kolatt, T. S., Kravtsov, A. V., Klypin, A. A., Porciani, C., \& Primack, J. R. 2001a, ApJ, 555, 240

Bullock, J. S., et al. 2001b, MNRAS, 321, 559 (B01)

Burstein, D., Bender, R., Faber, S., \& Nolthenius, R. 1997, AJ, 114, 1365

Caldwell, J. A. R., et al. 2005, preprint (astro-ph/0510782)

Christodoulou, D., Shlosman, I., \& Tohline, J. 1995, ApJ, 443, 551

Conselice, C. J., Bundy, K., Ellis, R. S., Brichmann, J., Vogt, N. P., \& Phillips, A. C. 2005, ApJ, 628, 160

Croton, D. J., et al. 2006, MNRAS, 365, 11

Dalcanton, J., Spergel, D., \& Summers, F. 1997, ApJ, 482, 659

Dekel, A., \& Silk, J. 1986, ApJ, 303, 39

Dutton, A. A., van den Bosch, F. C., Dekel, A., \& Courteau, S. 2007, ApJ, 654, 27

Efstathiou, G., Lake, G., \& Negroponte, J. 1982, MNRAS, 199, 1069

Eke, V. R., Navarro, J. F., \& Steinmetz, M. 2001, ApJ, 554, 114

Fall, S., \& Efstathiou, G. 1980, MNRAS, 193, 189

Ferguson, H. C., et al. 2004, ApJ, 600, L107

Flores, R., Primack, J., Blumenthal, G., \& Faber, S. 1993, ApJ, 412, 443

Giavalisco, M., Steidel, C., \& Macchetto, D. 1996, ApJ, 470, 189

Gnedin, O. Y., Kravtsov, A. V., Klypin, A. A., \& Nagai, D. 2004, ApJ, 616, 16

Gnedin, O. Y., Weinberg, D. H., Pizagno, J., Prada, F., \& Rix, H.-W. 2006, preprint (astro-ph/0607394)

\section{REFERENCES}

Governato, F., et al. 2004, ApJ, 607, 688

Häussler, B., et al. 2007, ApJS, 172, 615

Jesseit, R., Naab, T., \& Burkert, A. 2002, ApJ, 571, L89

Jing, Y. P. 2000, ApJ, 535, 30

Kassin, S. A., et al. 2007, ApJ, 660, L35

Kauffmann, G. 1996, MNRAS, 281, 475

Kauffmann, G., Colberg, J. M., Diaferio, A., \& White, S. D. M. 1999, MNRAS, 303,188

Kazantzidis, S., et al. 2005, ApJ, 623, L67

Klypin, A., Zhao, H., \& Somerville, R. S. 2002, ApJ, 573, 597

Kroupa, P. 2001, MNRAS, 322, 231

Lilly, S., et al. 1998, ApJ, 500, 75

Lowenthal, J., et al. 1997, ApJ, 481, 673

Macciò, A., Dutton, A., van den Bosch, F., Moore, B., Potter, D., \& Stadel, J. 2007, MNRAS, 378, 55

Maller, A. H., \& Dekel, A. 2002, MNRAS, 335, 487

Maller, A. H., Prochaska, J. X., Somerville, R. S., \& Primack, J. R. 2001, MNRAS, 326, 1475

Mao, S., Mo, H. J., \& White, S. D. M. 1998, MNRAS, 297, L71

Mo, H., Mao, S., \& White, S. 1998, MNRAS, 295, 319 (MMW98)

Natarajan, P. 1999, ApJ, 512, L105

Navarro, J. F., Frenk, C. S., \& White, S. D. M. 1997, ApJ, 490, 493

Navarro, J. F., \& Steinmetz, M. 2000, ApJ, 538, 477

Navarro, J. F., \& White, S. D. M. 1994, MNRAS, 267, 401

Peebles, P. J. E. 1969, ApJ, 155, 393

Pizagno, J., et al. 2007, AJ, 134, 945

Ravindranath, S., et al. 2004, ApJ, 604, L9

Rix, H.-W., et al. 2004, ApJS, 152, 163

Robertson, B., Bullock, J. S., Cox, T. J., Di Matteo, T., Hernquist, L., Springel, V., \& Yoshida, N. 2006, ApJ, 645, 986 
Robertson, B., Yoshida, N., Springel, V., \& Hernquist, L. 2004, ApJ, 606, 32

Sargent, M. T., et al. 2007, ApJS, 172, 434

Shen, S., et al. 2003, MNRAS, 343, 978 (S03)

Sheth, R. K., \& Tormen, G. 1999, MNRAS, 308, 119

Simard, L., et al. 1999, ApJ, 519, 563

Somerville, R., \& Primack, J. 1999, MNRAS, 310, 1087

Sommer-Larsen, J., Gelato, S., \& Vedel, H. 1999, ApJ, 519, 501

Spergel, D. N., et al. 2007, ApJS, 170, 377

Springel, V., \& Hernquist, L. 2005, ApJ, 622, L9
Syer, D., Mao, S., \& Mo, H. J. 1999, MNRAS, 305, 357

Trujillo, I., et al. 2004, ApJ, 604, 521

2006, ApJ, 650, 18 (T06)

van den Bosch, F. C. 2000, ApJ, 530, 177

Wechsler, R. H., Bullock, J. S., Primack, J. R., Kravtsov, A. V., \& Dekel, A. 2002, ApJ, 568, 52

Weil, M. L., Eke, V. R., \& Efstathiou, G. 1998, MNRAS, 300, 773

Wolf, C., et al. 2004, A\&A, 421, 913 